\title{
Investigating the gap between research and practice in additive manufacturing
}

Jennifer Bracken*, Zachary Bentley†, James Meyer†, Erik Miller†, Kathryn W. Jablokow‡, Timothy W. Simpson ${ }^{\ddagger}$ and Nicholas A. Meisel $¥$

* Department of Mechanical Engineering, Penn State, University Park, PA 16802

† Additive Manufacturing and Design Program, Penn State, University Park, PA 16802 ¥ School of Engineering Design, Technology, and Professional Programs, Penn State, University

Park, PA 16802

${ }^{¥}$ Department of Mechanical Engineering and Industrial Engineering, Penn State, University Park, PA 16802

\begin{abstract}
$\underline{\text { Abstract }}$
Additive manufacturing (AM) provides opportunities to design objects differently than traditional manufacturing methods allow, but only if designers understand the possibilities AM presents. In this study, we examined whether an AM workshop combined with an idea generation session could inspire engineering professionals to use AM solutions to solve current technical problems they face. All subjects were employees at an organization that will be referred to as Company X, a multinational commercial organization based in North America. During the study, we collected ideas for 24 projects generated before and after a training workshop focused on design for AM. In the workshop, we provided three hours of instruction about design for two metal-based AM processes. The participants' ideas were assessed using four specific metrics: (1) cost, (2) time, (3) completeness of solution, and (4) quality, which was a function of feasibility, usefulness, and novelty. Using these data, we explored whether the workshop was effective in inspiring the participants to use AM methods and techniques from AM research in their concept generation and whether participants' AM solutions showed improvement in cost, implementation time, and quality over non-AM designs generated before the workshop.
\end{abstract}

Additive Manufacturing, Design

Keywords:

\section{Introduction}

As a concept, additive manufacturing (AM) has generated considerable excitement in the product design and manufacturing domains, but putting AM into practice remains a challenge for some businesses [1]. Design for additive manufacturing (DfAM) presents different opportunities and restrictions than most traditional manufacturing methods, and these differences vary within the AM industry between machines and processes [2-4]. If these restrictions and opportunities are not well understood by the designers and engineers contemplating the use of AM, implementing the technology can look risky or expensive due to the long learning curve and high engineering cost. This paper explores recognizing AM design opportunities in corporate settings by discussing a study of a workshop to inspire design engineers to identify ways to use AM within a corporate setting. The business, which will be referred to as Company $\mathrm{X}$, examined during this study has invested millions of dollars in AM, and tasked numerous people with exploring the new technologies. They have extensive research efforts related to AM. However, only one AM part is currently used in Company X's production. Within Company X, many people have theories about why AM has not been more broadly implemented, ranging from "no one knows what to use it for" 
to "AM is not applicable to our projects." To date, however, no one has tested these theories with a cross-section of designers within the company. The current study was chartered to explore whether targeted DfAM education could lead employees to generate more design ideas that used AM, thus helping the business transition from using AM primarily for research to implementing AM technology more fully. Only one AM part had been put into production at Company X before this study, despite millions of dollars being invested in AM technology. To help Company X address their concerns about AM implementation, the current study involved several key activities:

1. gathering ideas generated as part of typical work at the company to use as baseline data,

2. holding an educational intervention followed by an idea generation session, and

3. comparing ideas gathered from before the educational intervention with those generated after the educational intervention.

This approach was aimed at giving the Company $\mathrm{X}$ designers enough information to move forward with AM designs and a chance to try brainstorming concepts for them. Idea generation was chosen for study because engineering designers' more detailed designs typically flow from concepts generated in this phase [3] and because the educational workshop is a standard technique for instilling new ideas to employees in Company X. Additionally, this study involved evaluation of the ideas generated to show how use of AM could affect time to implement, cost to implement, or quality of designs.

The rest of this paper elaborates on the study and analyzes the results. We begin with a discussion of related literature that informed and inspired the study design, followed by details of the study design. Next, data collection of baseline ideas generated before the AM education workshop, data collection of ideas generated after the AM education workshop, and data analysis are covered. This paper also presents key results of the study and their implications for industry (including Company X) and the AM research community. Finally, a brief discussion of study limitations and areas for future work concludes the paper.

\section{$\underline{\text { Relevant Literature }}$}

The study in this paper has been heavily influenced by previous research in the domains of AM, engineering design, and design education. First, it was important to review work that combined AM with design and idea generation, as this study intended to explore this combination. For example, Yang and Zhao [4] looked at Design for Additive Manufacturing (DfAM) with respect to conventional Design Theory and Methodology and how the two perspectives can be combined to produce better designs. Their review of design frameworks to be applied to AM in their paper inspired presenting design heuristics in the educational portion of the workshop conducted during our study.

A number of other studies have also examined how AM can be combined with idea generation or have described work that explores techniques for inspiring new concepts. For example, our study used the findings of Laverne et al. [5], a case study in which participants were given a design task and AM education. Laverne et al.'s found that introducing restrictive aspects of AM after the design process began was frustrating for participants. To alleviate that frustration in our study, we explained the restrictive aspects of AM to the participants before their idea generation began. 
Consequently, all of the information about DfAM was presented to the workshop participants before idea generation in this study. Additionally, positive or opportunistic aspects of DfAM (e.g., the ability to build complex geometry [6] or consolidate parts [7]) were presented as a larger portion of the educational material than negative or restrictive DfAM aspects (e.g., the need to worry about residual stress [8][9] or unsupported overhangs [9]). In this way, we hoped to mitigate any feelings of geometry-based design restriction during the design process.

Our workshop design also drew inspiration from work that was even more explicit about geometric guides for AM, such as the research of Maidin et al. [10], who ran trials on practitioners and students using a feature database as an approach to facilitate AM design. Their study concluded that their method was effective, although better results were found with students than with professionals. The success of Maidin et al.'s work led to the decision to include examples of successful dimensions for geometric features in the educational portion of the AM workshop conducted in our work.

While we focused heavily on design techniques, human subjects research also informed our study. In particular, the idea generation metrics created by Dean et al. [11] and Shah et al. [5][14] informed our assessment of design concepts, along with the work of Henderson et. al. [13]. All of these researchers used a selection of categories to breakdown quality into several components. The metrics in these papers, specifically, feasibility, usefulness, and novelty were chosen to assess quality in this study. Additionally, data collection techniques such as the spider plots used in [14] were implemented to reduce survey fatigue in subjects. Using this work, we evaluate how well the workshop's AM education improved idea generation from the baseline idea generation before the workshop. For the idea generation segment of the study, methods suggested in previous work by Watschke et al. were employed. Specifically, Watschke at al. [15] developed and evaluated several different ideation methods. They scored them on suitability to relate them to AM and ranked them according to how much knowledge of the method was required to use the method. Based on Watschke et al.'s findings, a combination of Brainstorming and Reizbild methods were selected in the current study, since users did not need extensive training to implement either method; this allowed workshop participants to focus on their designs and not the idea generation techniques. These methods were implemented to help stimulate the ideation process by displaying models and images of additively manufactured parts at each table.

In addition to using models, images, and specific methods of idea generation, AM techniques drawn from the DfAM literature were featured in our workshop training materials. For instance, part consolidation, or combining multiple components into fewer components, is a design technique that is uniquely suited to AM due to the complexity it enables [9][18][19]. Other techniques discussed included topology optimization [18] and inventory reduction [19]. Topology optimization makes use of AM's ability to create complex structures. Inventory reduction was included as another topic of interest to Company X, as they always keep many parts in stock. Examples of AM part designs from industry and academia were included during the workshop in order to inspire participants to think about creative geometries and ways AM can induce cost savings [20]. All of this information was presented to help show the relevance of AM to Company $\mathrm{X}$, and to inspire ideas to leverage the opportunities AM presents. 


\section{$\underline{\text { Research Ouestions }}$}

As mentioned previously, techniques successful for other studies were combined in hopes of seeing design solutions that employed AM emerge during idea generation. However, generating AM solutions was not the sole goal of the study, as Company $\mathrm{X}$ also wished to see the generated ideas improve in specific ways that indicated they would add value in key areas, not simply provide research projects. These key areas included cost to implement, time to implement, and quality of design. Additionally, the training workshop was designed to increase the number of design solutions that utilized AM capabilities and improved quality, while decreasing time and cost to implement those designs. In order to examine the influence of the workshop on these solution characteristics, the team posed four research questions (RQs):

1) How will a one half-day introduction to AM training workshop affect the number of AMbased design ideas generated in response to the problem set?

2) How will AM solutions generated after the workshop score, according to expert raters, compared to baseline designs with respect to quality?

3) How will AM solutions generated after the workshop score, according to expert raters, compared to baseline designs with respect to speed of implementation?

4) How will AM solutions generated after the workshop score, according to expert raters, compared to baseline designs with respect to engineering cost?

We hypothesized that having an AM training workshop immediately before the idea generation session would increase the number of generated design ideas that use AM. This was anticipated because people would have just been exposed to information about opportunities to design using $\mathrm{AM}$ and why it is useful. It was also expected that some of these new designs would be of higher quality, faster to implement, and less expensive based upon examples of AM solutions throughout the literature, as mentioned in the literature review. Details on the experimental methods follow.

\section{Experimental Methods}

To answer the research questions and test the hypotheses, a study was designed to include a training workshop about DfAM followed by an idea generation session in which participants were tasked with proposing solutions to existing projects for the business. Design ideas were collected before the training workshop was held, and again after the idea generation session to allow for comparison. Both sets of ideas were rated by expert reviewers for consistency. The resulting data were analyzed by the research team. Figure 1 diagrams the flow of the experimental process, and the next section discusses this experimental method in more detail.

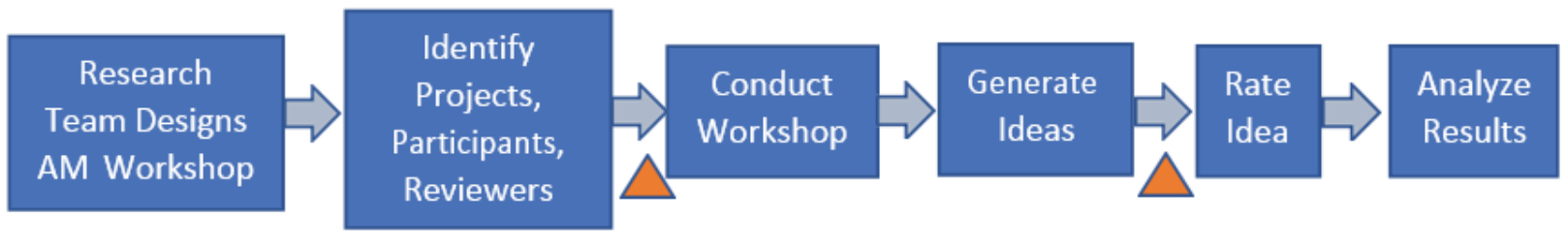

Figure 1: Flow of the experimental process. Triangles represent when ideas were gathered/collected. 
The structure of the workshop was designed so that participants would see how AM could be useful for the business in ways beyond providing research opportunities. The half-day AM training workshop consisted of an introduction, guided discussion, and three lecture-style periods broken up by breaks and short, interactive breakout sessions to encourage group discussion and help cement the concepts learned. This flow of the training workshop can be seen in Figure 2.

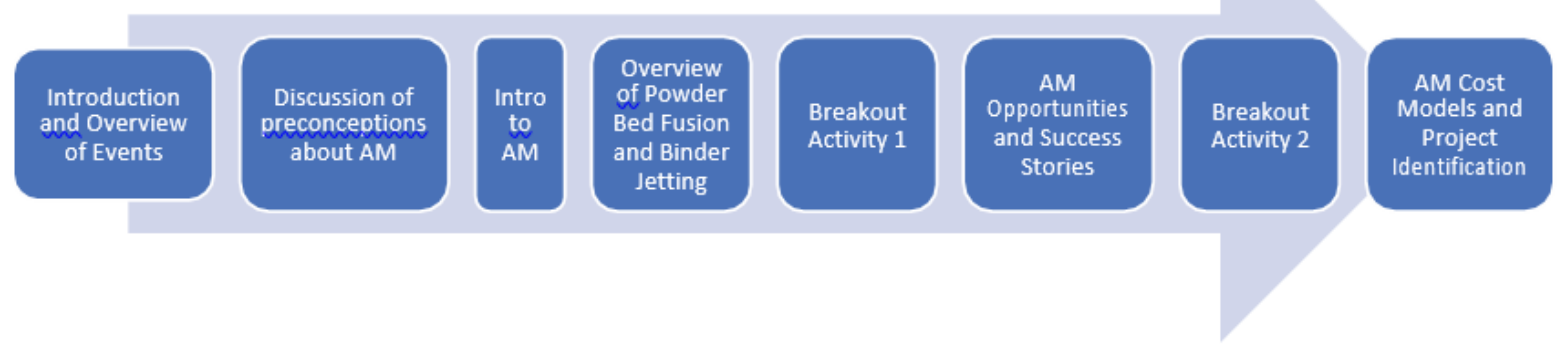

Figure 2: Flow of training workshop about Additive Manufacturing.

Following the AM training workshop, participants were randomly divided into teams of four or five people. Each team was provided with three project prompts. The teams were then given 90 minutes to generate solution ideas for each of the three projects. They were provided with idea sheet templates on which to record their ideas.

\section{Design of the AM Education Workshop}

To keep the workshop focused, the AM training workshop included only Company X's two most readily available metal-based AM modalities, laser-based Powder Bed Fusion and Binder Jetting. A brief outline of the lecture-style content follows:

- Fundamental AM concepts and a brief overview of additive manufacturing in general

- Process overviews of Powder Bed Fusion (PBF) and Binder Jet modalities, with focus on the machine models currently available, along with fundamentals of post-processing steps

- Restrictive considerations for PBF (supports, avoiding trapped powder, post processing, size limitations, etc.) and Binder Jetting (size, trapped powder, shrinkage, etc.)

- AM opportunities (geometric complexity, light-weighting, part consolidation, design time to implement reduction, supply chain disruption, etc.)

- Examples of success stories (from other businesses, but focusing on the industry of which Company $\mathrm{X}$ is a part)

- How to build a business case for an AM design (project identification, cost modeling, supply chain benefits, growth opportunities, performance benefits, etc.)

Additionally, a target opportunistic-to-restrictive lecture content ratio for the AM instruction workshop was set at 2:1 in order to focus more on inspiration and positive aspects of AM than on the challenges of AM. This target ratio was selected to promote a tempered sense of design freedom related to AM, rather than one of being restricted by process limitations of AM, or an unrealistic one of being able to manufacture anything using AM. Literature from previous work of two of the authors reinforces the importance of teaching both restrictive and opportunistic aspects of AM technologies [21-22]. The actual time spent on each topic met this time target 
within 5 minutes (roughly 40 minutes opportunistic to 20 minutes restrictive). Opportunistic content was purposely presented after the restrictive content, so that participants would feel inspired and motivated going into the idea generation session [23]. Figure 3 illustrates the fractional time spent on each type of activity, breaking down the lecture period further by the type of content presented.

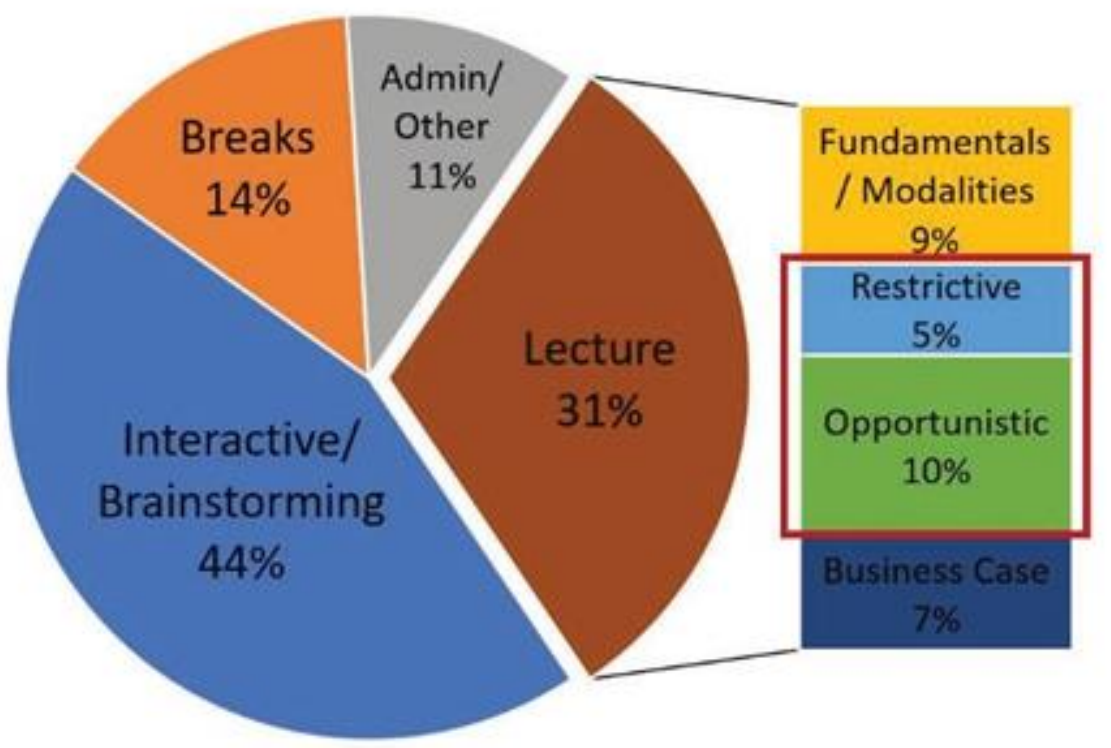

Figure 3: Study time allotments by topic. The workshop took up half of a workday (4 hours). All activities combined, including the 1.5 hour idea generation session, took 6.5 hours.

The training content relied heavily on imagery and physical examples to communicate ideas. Over 70 photographs of various AM printed parts were included in the lecture material. Three short videos were also shown to better illustrate the metal AM processes. More than a dozen additively-manufactured metal parts were passed around during the session as examples of both successful designs, such as an intricate heat exchanger, and designs that did not print well, such as residual-stress-warped components. Additionally, two handouts were provided to each team prior to the idea generation session: one handout highlighted general restrictive design guidelines for each process (including minimum feature size, overhang angle, support recommendations, etc.), and the other containing 12 photographs of opportunistic AM geometries to provide inspiration, such as part consolidation examples and very complex lattice structures that had been printed in metal.

Finally, to reiterate the notion that AM can be a realistic solution for Company X, a design engineer who had worked on an AM project (and who was also a workshop participant) was asked to speak about his project for 10 minutes during the "success stories" portion of the lecture. Two other participants who had some limited experience with other AM projects (one metal, one polymer) volunteered their insights and positive feedback to the entire group, unprovoked. These "peer testimonials" likely lent further credibility and context for AM in the business, as peer recommendations have been found to be a persuasive tool in other studies [24].

Another important aspect of this experiment was selection of appropriate projects for which to 
generate solutions during the idea generation session. Twenty-four different projects were selected for this study's idea generation session, from a pool that Company X's reliability group had tracked and addressed. Projects with solutions already designed were selected preferentially, in order to allow comparison of any new concepts generated during the study with designs generated as part of typical work at the company using non-AM techniques. Specifically, projects were chosen that had a proposed current solution, a root cause identified for each problem, a time estimate for implementation, and an implementation cost estimate. The time investment the company had in these projects before the study showed the criticality they had to the business and its customers, which made the workshop more meaningful to the business and the workshop participants. Additionally, each of the selected projects was mechanical in nature, since the workshop focused on building mechanical components with AM

\section{Participants}

The workshop participants included 34 mechanical design engineers employed at Company X. These engineers had a wide range of experience in the engineering disciplines represented in the chosen workshop projects. They also had a varying number of years of engineering industry experience, with an average of 16.3 years (see Figure 4). The engineers were randomly assigned to eight teams with four or five participants per team. Four of the selected engineers were the original leads for the projects selected, although this was not used to assign projects to teams. There were concerns that having engineers who had worked on the projects previously could cause design fixation [25]-[27]. However, to make the study as accurate a representation of the design process for Company $\mathrm{X}$ as possible, using the typical design engineers for the workshop was felt to be important. The importance of using the correct employees was felt to outweigh any concerns about design fixation, provided designs were reviewed and checked to make sure they were not the same.

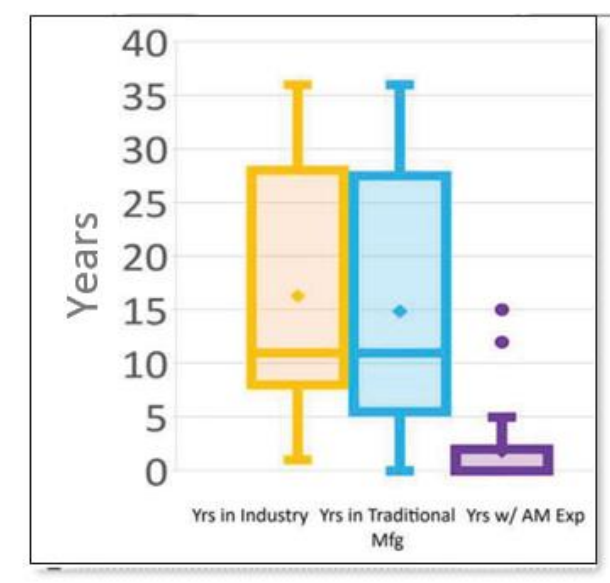

Figure 4: Plot of years participants spent in industry, traditional manufacturing, and with AM.

The level of AM knowledge was not explicitly considered when selecting the engineers for the workshop, as it was believed to be generally low across the company. This assumption was validated by a brief survey of the participants taken at the beginning of the AM instruction workshop, in which it was found that most had minimal knowledge of AM, and only had experience with AM recently, as expected. The results of that survey, during which participants' years in traditional manufacturing and years in industry were also collected, can be seen in Figure 
4. Average years in traditional manufacturing was 14.9 years, and in stark contrast experience, with Additive Manufacturing averaged only 1.8 years, with many individuals answering 0 years.

\section{Idea Generation Session}

The idea generation session using these projects was held directly after the educational workshop. The projects selected were randomly split into groups of three to be given to the eight different teams of engineers, so that each project was only examined by one group of engineers and had one proposed solution before and after the workshop. Each team was given three idea sheets on which to record their ideas. The idea generation session was 90 minutes long. In order to collect data that better reflected how useful the participants found the AM technology, it is important to note that there was no requirement for solutions generated during the idea generation session to use AM. This was explicitly stated when the session began, by the facilitator.

\section{Idea Reviewers}

The expert reviewers who rated the ideas generated by the workshop participants were chosen from among Company X's most senior principal engineers. Two of the reviewers have mechanical backgrounds, and one has a materials-based background; they average 26.3 years of design experience. These experts routinely review and evaluate engineering designs as part of their roles within the business. While they do not explicitly focus on AM technologies as part of their dayto-day expertise, they completed a two-day AM training session (independent of this study) approximately three weeks before our study's AM training workshop and idea generation. The two-day AM training session was led by an external training firm specializing in additive manufacturing and focused on Laser Powder Bed Fusion and Binder Jetting. This added training, combined with these engineers' decades of experience in assessing engineering designs, was considered adequate qualification to rate the designs produced in the workshop.

\section{Metrics}

The reviewers were tasked with evaluating the 24 pre-workshop solution designs using the following factors: cost, completeness of solution, time to implement solution, and quality. Quality was broken down further into three dimensions - Feasibility, Usefulness, and Novelty — via spider plots, as shown in Figure 5. These dimensions of quality align with the idea generation metrics referenced earlier [3][11][12][14]. Novelty is intended to be a measure of how unique the idea is compared to all other ideas the reviewers have seen across their experiences with engineering. Feasibility is intended to be a measure of whether or not the idea is realistic and consumes reasonable resources. Usefulness is intended to be a score of how helpful the idea will be to solve the problem. The three scores for each idea in Feasibility, Usefulness, and Novelty were averaged to create an overall Quality score for each idea, and thus make quality less subjective. 


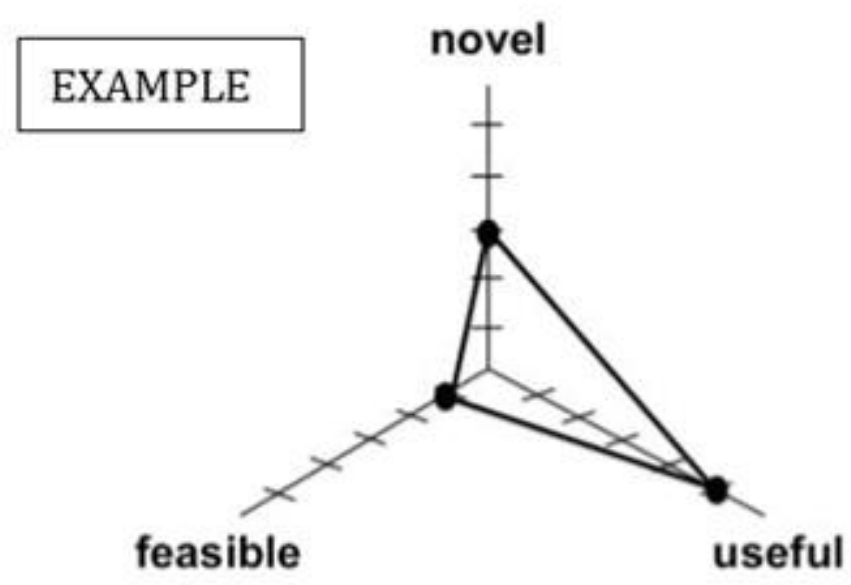

Figure 5: Diagram of Example Spider Plot Showing Quality Sub-Dimensions

The other questions in the reviewers' scoring sheets used worded answers associated with numbers to rank cost, time, and completeness of solution. Cost of each solution could be scored as 1) Thousands, 3) Hundreds of Thousands, or 9) Millions. Time to implement each solution could be scored as 1) Weeks, 3) Months, or 9) Years. These dramatic differences between cost and time categories were the reason for selecting non-linear scales of 1, 3, 9. This nontraditional scale was previously utilized for metrics based on the Quality Function Deployment (QFD) method QFD [28]. The goal in QFD is to distinguish between ideas that could be considered very important and those that are trivial. In this study, we believe this nonlinear scale will provide raters with clear distinctions between "high" and "low" scores

Linear scales were used for completeness and the previously discussed three dimensions of quality. Completeness was rated using a linear 1-3 scale. Scores included: 1) Does not solve the problem, 2) Partially solves the problem, and 3) Completely solves the problem. For each subcategory of quality, raters used a 1 to 5 scale, with 5 being the best in each instance. No words were associated with those answers. Spider plots were used for these ratings to reduce survey fatigue, as in previous work by some of the authors [29]. The ratings of the pre-workshop solution ideas became the baseline data to which the ratings for the ideas generated during the workshop were compared.

After the workshop, the same expert reviewers were asked to score the AM-based solutions proposed by the teams during the idea generation session. For this rating process, they used the same worksheets, criteria, and scales that were used for the baseline solution ideas. They worked together to identify scores for each team as a group because that is how they usually work together, and they felt that they could best combine their expertise in that manner. This is a limitation of this study, as having them score designs individually would have been better because inter-rater reliability could have been examined. The designs were also reviewed (but not scored) by the company's AM team and members of the research team to check for any unusual results, such as teams who did not specify how products would be manufactured. Only two teams failed to specify this clearly, and the researchers followed up with them to clarify their designs.

\section{Data Collection}


The research team gathered pre-workshop design solutions for all 24 of the projects examined. These solution designs were generated before the study as part of typical work by engineers at the company, not by workshop participants. Workshop participants were not shown these solutions during the later workshop, in order to avoid design fixation. All of the collected designs were transcribed onto a standard template for analysis. Included in that template were three items: 1) the solution idea/design as a sketch or CAD with a written description, 2) its approximate timeline for implementation, and 3) estimated engineering cost to implement. A sample idea template can be seen in Appendix A. These pre-workshop ideas were used as a baseline to help reviewers identify whether ideas generated during the proposed workshop were better in terms of cost, time, solution completeness, or quality than those generated before it. In total, data was collected in the categories seen in Table 1. After the AM instruction workshop, the participating teams generated new solutions for all 24 projects. Two of those ideas will be briefly reviewed here to provide examples of typical projects. Scores for each example can also be seen in Table 1.

Table 1: Metrics used to assess the ideas, and scores of both example projects.

\begin{tabular}{|l|l|l|}
\hline Idea Metrics & Scores for Team 2 Example & Scores for Team 6 Example \\
\hline Feasibility Rating & 3 & 2 \\
\hline Usefulness Ratings & 4 & 3 \\
\hline Novelty Ratings & 4 & 3 \\
\hline Quality Ratings & 3.67 & 2.67 \\
\hline Satisfaction Ratings & 3 & 2 \\
\hline Time to Implement & 9 & 9 \\
\hline Cost to Implement & 3 & 9 \\
\hline Completeness of Solution & 2 & 2 \\
\hline
\end{tabular}

The first example comes from Team 2, who proposed a unique solution to a field reliability problem where a standard cooler is slightly undersized for the temperature and altitude requirements of the project. The undersizing affects the performance of the product, as the machine reduces horsepower when the cooling requirements cannot be met. A bigger cooler would require repackaging the entire machine and is therefore impractical. Their proposed solution took advantage of the free complexity offered by additive manufacturing. They proposed that the cooler could be made more efficient by increasing the internal passageway surface area using a spiral tube in a pre-cooling stage, made using binder jet technology. An excerpt from their idea sheet is shown in Figure 6.

Team 2's idea scored 3.67 in overall quality, with scores of 4 for novelty, 3 for feasibility, and 4 for usefulness, making it a high scoring idea among those seen in this workshop. Team 2's idea scored 2 (or "partially meets outcomes") for how completely the solution solved the problem. It received a customer satisfaction rating of 3/4 (somewhat satisfied), and it received a time to implement rating of $9 / 9$ (which means it was expected to take a long time to implement.) Cost was scored as 3, which means it cost less than most solutions scored in this study, as the average costs of both pre-workshop ideas and post-workshop ideas were both higher than 3 . To compare these scores to the average team scores for cost, see Table 2. From both of these worksheets, 
which were typical results, it is evident that these two teams understood the lecture content well and applied it. Both teams successfully leveraged different aspects of opportunistic AM, as intended by the workshop.

Second, Team 6 was assigned to look at a reliability issue on the hot side of an engine pipe. They proposed a new part that reduced part count and added functionality, adding bellows to accommodate the thermal expansion and other motions. They maintained the same mechanical interface for backward compatibility and theorized that a non-constant cross section would minimize back pressure. In Figure 7Figure, the original design (proposed by other engineers prior to the workshop, not Team 6) can be seen on the left, and the design proposed by Team 6 is on the right. This team also took advantage of Company X's 3D CAD model library. The proposed solution included design, analysis, and validation estimates, as well as cost estimates. This idea scored 2.67 in overall quality, with scores of 3 for novelty, 2 for feasibility, and 3 for usefulness; this value is fairly typical for this workshop, where the average overall quality rating for AM ideas as 3.03. The new solution of Figure 7 scored 2, or "partially meets outcomes" for how completely the solution solved the problem, and it received a customer satisfaction rating of 2/4 (somewhat satisfied) and a time to implement rating of 9/9 (which means it was expected to take a long time to implement.) Cost was also scored as a 9, which means it was expected to be an expensive solution. To compare these values to the average scores of all ideas gathered and collected during this study see Table 2.

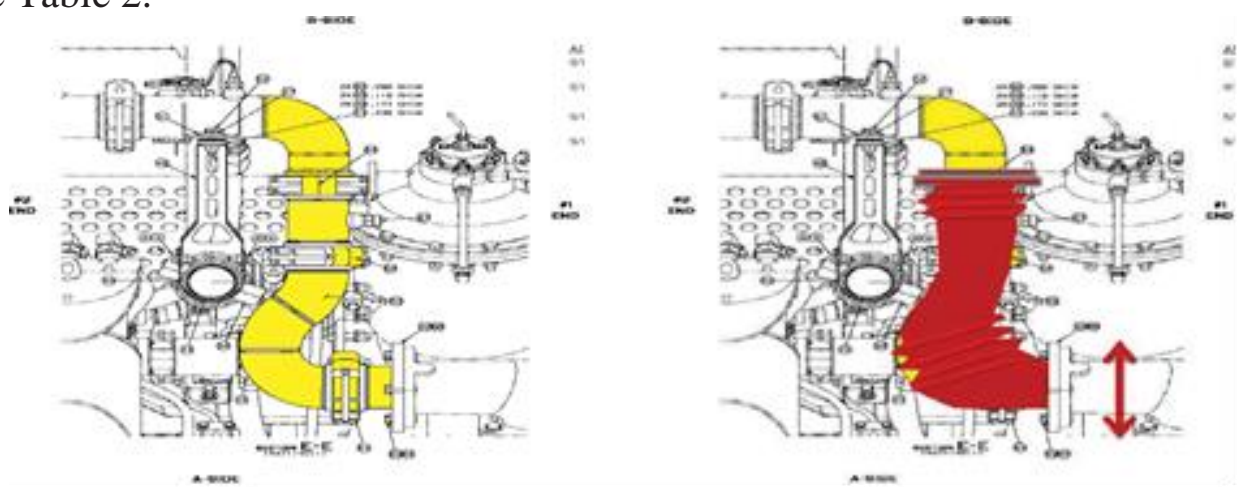

Figure 7: Team 6, Original concept (left) and new additive proposed concept (right).

\section{Analvsis and Results}

Once all of the data were collected, they were compiled in a spreadsheet to perform statistical analyses. All data sets were normally distributed except for solution completeness, and thus, they could be assessed using paired t-tests in order to compare ideas generated before the AM workshop with ideas generated after the AM workshop. However, because the solution completeness data set was not normally distributed, a different test was employed to analyze those data, as elaborated later in this section. Results of the data examined using t-tests can be seen in Table 2. 
Table 2: P-Values and Average Ratings of the Ideas Generated Before and After the Workshop. $P$ values are generated by performing t tests. A 95\% level of confidence was used for all tests.

\begin{tabular}{|l|c|c|c|}
\hline $\begin{array}{l}\text { Idea Metric Compared } \\
\text { Before and After } \\
\text { Workshop }\end{array}$ & $\begin{array}{c}\text { Average of Pre- } \\
\text { Training Ideas } \\
\text { (all non-AM) }\end{array}$ & $\begin{array}{c}\text { Average of AM } \\
\text { Ideas After } \\
\text { Training }\end{array}$ & P value \\
\hline Feasibility Rating & 3.18 & 2.55 & 0.076 \\
\hline Usefulness Ratings & 2.64 & 3.45 & 0.041 \\
\hline Novelty Ratings & 1.64 & 3.09 & 0.00045 \\
\hline Quality Ratings & 2.48 & 3.03 & 0.023 \\
\hline Satisfaction Ratings & 2.45 & 2.45 & 0.500 \\
\hline Time to Implement & 3.00 & 4.45 & 0.030 \\
\hline Cost to Implement & 5.18 & 6.82 & 0.079 \\
\hline
\end{tabular}

The results found during the analysis were promising for RQ 1 , as the increase in teams using $\mathrm{AM}$ as part of their solution after the workshop suggested that the training workshop was an effective intervention to inspire people to generate solution ideas that use AM. In the preworkshop idea pool, no ideas used AM at all. There was a possibility that the pre-workshop ideas could include AM, due to the business' excitement about AM, but because of the low rate of AM adoption throughout the business, the lack of AM ideas was not surprising. However, after the workshop, 11 out of 24 ideas employed AM in some manner. Additionally, RQ 2, which was about how quality of AM designs would perform after the workshop, had a positive result. Overall quality ratings for the $11 \mathrm{AM}$ ideas were statistically higher on average than pre-workshop nonAM solutions to the same problems. This indicates that participants were not only coming up with ideas that used AM, but ideas that could add value to the business. P-values for the t-tests are shown in Table 2. The score categories of Novelty, Usefulness, and Quality all showed improvement in average score of AM ideas generated after the workshop, via higher mean scores than their non-AM counterparts gathered before the workshop. Feasibility scores remained about the same, as can be seen in Table 2 .

When examining RQ 3, which considered how cost was affected, it can be seen in Table 2 that the cost to implement scores were not statistically different for the solutions generated before and after the workshop (i.e., these scores were effectively the same on average for both the workshopbased AM ideas and the pre-workshop non-AM ideas). Meanwhile, for RQ 4, or time to implement solutions, the outcome of the data analysis was not as supportive of AM as expected. The Time to Implement of the 11 AM ideas was not statistically equivalent for the projects before and after, but it was statistically longer for the 11 AM ideas than their non-AM counterparts. This implies that the expert raters believed the AM ideas would take somewhat longer to implement on average than the non-AM ideas generated before the workshop. The system the raters used to rate these ideas in such categories did not require explanations of why ideas were expected to take longer, so whether or not the longer times to implement were the product of an inherent bias against AM is unclear at this time. To explore how time is affected by AM designs more effectively in future work, we will investigate using detailed quotes of time to implement for all ideas, instead of the rating systems used in this work. 
In addition to the data specifically related to the four RQs, completeness of solution data were collected and analyzed in order to track whether designs were solving problems in their entirety. These data were not normally distributed; so, a Wilcoxon test was used to assess them. This was done at a $95 \%$ level of significance, but on only 10 of the paired ideas from before and after the workshop. Only 10 could be compared because our reviewers stated that they were unable to assess one idea for this category because they did not have enough information. The p-value for this test was 1, which indicates that the two sample means were not statistically different. Thus, we saw no significant difference in completeness of solution between non-AM solutions generated before the workshop and AM solutions generated after the workshop for the same project. This suggests that ideas that did not use AM before the workshop were equivalently complete solutions as those using AM after the workshop. This is very interesting to see, as the pre-workshop nonAM solutions were laid out as part of the normal workflow of the company and planned for implementation; so, it was expected that those solutions would have solved the problems more completely. This result indicates that workshop participants took the idea generation session very seriously, as they were able to generate solutions that solved the problems approximately as effectively as the solutions that were generated before the workshop as part of normal business.

\section{Conclusions, Limitations, and Future Work}

The results of the study are promising for training workshops paired with idea generation sessions encouraging designers to employ AM during idea generation. It appears that duration of the workshop attendance was enough for engineers to propose ideas that employed AM, which was the main question the team sought to answer. Additionally, it is notable that the quality of the ideas improved significantly between the non-AM ideas and the AM ideas. This suggests future work with AM is worth exploring further for the company. It significantly increased novelty and usefulness particularly, which could be very useful from an intellectual property standpoint.

There were several limitations of this work that may affect the results. Firstly, the expert raters worked as a team. This means that it is possible that one dominant member of the group may have swayed decisions, and it means average scores are unavailable. To avoid this in the future, expert raters should not be allowed to communicate during scoring. That will allow for comparison of scores and inter-rater reliability assessments. Additionally, time and cost estimations of the AM ideas should be further explored in the future, as the educational portion on those topics could be improved to facilitate idea performance gains. It appeared that ideas examined here were not expected to show performance improvement in time and cost, though literature suggests AM can show improvements in those categories [19][20][30]. Finding specific and accurate cost and time quotes for each AM idea would help with this process in the future, but it would require extending studies to include time for more detailed designs and quotes. Another topic that should be explored in the future is how the non-AM ideas proposed after the workshop compared to the ideas proposed before the workshop. There was not enough time budgeted for the expert raters to review all of the non-AM ideas in depth after the workshop; so, it is not possible at this time to discuss whether these ideas all showed consistent improvements even if they did not use AM. If more expert rater time could be allotted in the future, then the idea of a workshop about AM with team idea generation could be explored as a general intervention to improve idea generation, and the ideas generated after the workshop could be compared to each other using AM and not using AM. 


\section{$\underline{\text { References }}$}

[1] I. Gibson, D.W. Rosen, B. Stucker, Additive Manufacturing Technologies: 3D Printing, Rapid Prototyping, and Direct Digital manufacturing, 2nd., Springer, New York, pp. 1$498,2015$.

[2] M. Kumke, H. Watschke, and T. Vietor, "A new methodological framework for design for additive manufacturing," Journal of Virtual and Physical Prototyping, vol. 11, no. 1, pp.3-19, February 2016.

[3] J. J. Shah, S. V. Kulkarni, and N. Vargas-Hernandez, "Evaluation of Idea Generation Methods for Conceptual Design: Effectiveness Metrics and Design of Experiments," Journal of Mechanical Design, vol. 4, no. 122, pp. 377-384, 2000.

[4] S. Yang and Y. F. Zhao, "Additive manufacturing-enabled design theory and methodology: a critical review," International Journal of Advanced Manufacturing Technology, vol. 80, no. 1-4. pp. 327-342, September 2017.

[5] F. Laverne, F. Segonds, N. Anwer, and M. Le Coq, "Assembly Based Methods to Support Product Innovation in Design for Additive Manufacturing: An Exploratory Case Study," Journal of Mechanical Design, vol. 137, no. 12, p. 121701, October 2017.

[6] B. P. Conner, G. P. Manogharan, A. N. Martof, L. M. Rodomsky, C. M. Rodomsky, Jordan, D. C. Limperos, W. James, "Making sense of 3-D printing: Creating a map of additive manufacturing products and services," Additive Manufacturing, vol. 1, pp. 64-76, October 2014.

[7] S. Yang, Y. Tang, and Y. F. Zhao, "A new part consolidation method to embrace the design freedom of additive manufacturing," Journal of Manufacturing Processes, vol. 20, pp. 444449, October 2017.

[8] L. Parry, I. A. Ashcroft, and R. D. Wildman, "Understanding the effect of laser scan strategy on residual stress in selective laser melting through thermo-mechanical simulation," Additive Manufacturing, vol. 12, pp. 1-15, October 2016.

[9] A. S. Wu, D. W. Brown, M. Kumar, G. F. Gallegos, and W. E. King, “An Experimental Investigation into Additive Manufacturing-Induced Residual Stresses in 316L Stainless Steel," Metallurgical and Materials Transactions A: Physical Metallurgy and Materials Science, vol. 45, no. 13, pp. 6260-6270, Oct. 2017.

[10] S. Bin Maidin, I. Campbell, and E. Pei, "Development of a design feature database to support design for additive manufacturing," Assembly Automation, vol. 32, no. 3, pp. 235244, July 2012.

[11] D. L. Dean, J. M. Hender, T. L. Rodgers, and E. L. Santanen, "Identifying quality, novel, and creative ideas: Constructs and scales for idea evaluation," Journal of the Association for Information Systems, vol. 7, no. 10, pp. 646-699, 2006.

[12] J. J. Shah, S. M. Smith, and N. Vargas-Hernandez, "Metrics for measuring ideation effectiveness," Design Studies, vol. 24, no. 2, pp. 111-134, 2003. 
[13] D. Henderson, K. Jablokow, S. Daly, Seda McKilligan, E. Silk, and J. Bracken, "Comparing the Effects of Design Interventions on the Quality of Design Concepts as a Reflection of Ideation Flexibility," Journal of Mechanical Design, vol. 141, no. 3, p. 031103, 2019.

[14] D. Henderson, A. Vora, K. Jablokow, J. Bracken, and N. Sonalkar, "Beyond Likert Scales: Exploring Designers' Perceptions through Visual Reflection Activities," in American Society of Engineering Education, June 2019, Tampa, Florida.

[15] H. Watschke, A.-K. Bavendiek, A. Giannakos, and T. Vietor, "A Methodical Approach to Support Ideation for Additive Manufacturing in Design Education," in Proceedings of 21st International Conference On Engineering Design, 2017, editors A. Maier, S. Skec, H. Kim, Michael Kokkolaras, Josef Oehmen, Georges Fadel, Filippo Salustri, Mike Van der Loos, p. 041-050.

[16] S. Yang, F. Santoro, and Y. F. Zhao, "Towards a Numerical Approach of Finding Candidates for Additive Manufacturing-Enabled Part Consolidation," Journal of Mechanical Design, vol. 140, no. 4, p. 041701, 2018.

[17] S. Yang and Y. F. Zhao, "Additive Manufacturing-Enabled Part Count Reduction: A Lifecycle Perspective,” Journal of Mechanical Design, vol. 140, no. 3, p. 031702, Jan. 2018.

[18] T. Zegard and G. H. Paulino, "Bridging topology optimization and additive manufacturing," Structural and Multidisciplinary Optimization, vol. 53, no. 1, pp. 175-192, 2016.

[19] W. E. Frazier, "Metal additive manufacturing: A review," Journal of Materials Engineering and Performance, vol. 23, no. 6, pp. 1917-1928, 2014.

[20] E. Atzeni and A. Salmi, "Economics of additive manufacturing for end-usable metal parts," International Journal of Advanced Manufacturing Technology, vol. 62, no. 9-12, pp. 1147$1155,2012$.

[21] R. Prabhu, S. R. Miller, T. W. Simpson, and N. A. Meisel, "The Earlier the Better? Investigating the Importance of Timing on Effectiveness of Design for Additive Manufacturing Education," in Proceedings of the ASME 2018 International Design Engineering Technical Conferences and Computers and Information in Engineering Conference, At Quebec City, Canada, August 2018.

[22] R. Prabhu, S. R. Miller, T. W. Simpson, and N. A. Meisel, "Teaching Design Freedom: Exploring the Effects of Design for Additive Manufacturing Education on the Cognitive Components of Students' Creativity," in Proceedings of the ASME 2018 International Design Engineering Technical Conferences and Computers and Information in Engineering Conference, At Quebec City, Canada, August 2018.

[23] M. Baas, C. de Dreu, and B. A. Nijstad, "Emotions that associate with uncertainty lead to structured ideation," Journal of Emotion, vol. 12, no. 5, pp. 1004-1014, Oct. 2018.

[24] Nielsen Holdings, "Global Trust in Advertising Report: Winning Strategies for an Evolving Media Landscape," Nielsen Insights, vol. 1, no. September 2015, pp. 1-22, 2018.

[25] J. S. Linsey, I. Tseng, K. Fu, J. Cagan, K. L. Wood, and C. Schunn, “A Study of Design Fixation, Its Mitigation and Perception in Engineering Design Faculty," Journal of Mechanical Design, vol. 132, no. 4, p. 12, April 2010. 
[26] A. T. Purcell and J. S. Gero, "Design and other types of fixation," Journal of Design Studies, vol. 17, no. 4, pp. 363-383, October 1996.

[27] D. G. Jansson and S. M. Smith, "Design fixation," Journal of Design Studies, vol. 12, no. 1, pp. 3-11, January 1991.

[28] C. P. M. Govers, "What and how about quality function deployment (QFD)," Journal of International Journal of Production Economics, vol. 46, pp. 575-585, December 1996.

[29] J. Bracken, F. X. Glavier, D. Henderson, K. Jablokow, N. Sonalkar, and A. M. Erdman, "Can Process Metrics Predict Product Success? A Pilot Study of Student Design Teams," in International Design Engineering Technical Conferences \& Computers and Information in Engineering Conference 2019, Anaheim CA, USA, August 2019.

[30] T. Kellner, "An Epiphany Of Disruption: GE Additive Chief Explains How 3D printing Will Upend Manufacturing," GE Reports, Boston, MA, USA, November 13, 2017. 